\title{
Evaluation of Renal Histopathological Changes, as a Predictor of Recoverability of Renal Function Following Pyeloplasty for Ureteropelvic Junction Obstruction
}

\author{
Kaushal Kumar, ${ }^{1, *}$ Ahsan Ahmad, ${ }^{1}$ Shailendra Kumar, ${ }^{1}$ Vijyanand Choudhry, ${ }^{2}$ Rajesh Kumar Tiwari, ${ }^{1}$ \\ Mahendra Singh, ${ }^{1}$ and Mohammad Ali Muzaffar ${ }^{1}$ \\ ${ }^{1}$ Department of Urology, Indira Gandhi Institute of Medical Sciences, Patna, India \\ ${ }^{2}$ Department of Pathology, Indira Gandhi Institute of Medical Sciences, Patna, India \\ "Corresponding author: Kaushal Kumar, Department of Urology, Indira Gandhi Institute of Medical Sciences, Patna, India. Tel: +91-9431457765, E-mail: \\ surgeonkaushal@rediffmail.com \\ Received 2015 March 07; Revised 2015 April 11; Accepted 2015 April 12.
}

\begin{abstract}
Background: Pyeloplasty is a widely accepted treatment for ureteropelvic junction obstruction(UPJO). However, the renal function recoverability after pyeloplasty is still a matter of debate. Different parameters have been used to predict renal functional recoverability after corrective surgery, with conflicting results.

Objectives: In this study, renal biopsy was carried on a series of cases of UPJO, during pyeloplasty, to study the extent of histological alterations in renal parenchyma, as a result of obstruction, and its predictive value in renal function recoverability after pyeloplasty. Patients and Methods: We retrospectively analyzed the renal biopsy obtained during pyeloplasty in 53 adult patients. Histopathological changes were graded on a scale of 1 to 3, according to their severity, and compared with the differential renal function (DRF) revealed on the preoperative and postoperative follow up diethylene triamine pentaacetic acid (DTPA) renal scan. A Fischer's t test was used to evaluate statistical differences between values.

Results: This study showed a linear relationship between the severity of histological changes and renal function recovery, after pyeloplasty. Out of 24 obstructed renal units (ORU), with minimal histopathological changes (grade I), 21 ORU ( $87.5 \%$ ), with $>35 \%$ DRF preoperatively, showed significant improvement in renal function after 12 months of pyeloplasty $(\mathrm{P}<0.05)$. On the other hand, all kidneys ( $n=29$ ) with moderate to severe obstructive changes (grade II and III) had minimal improvement in DRF, after pyeloplasty, which was clinically insignificant $(\mathrm{P}>0.05)$. Renal function deterioration after pyeloplasty was not observed in any of the cases. Conclusions: The severity of pathological changes in renal parenchyma, due to UPJO, is a good predictor of renal function recoverability, after pyeloplasty. The ORUs, with DRF > 35\%, usually have normal (grade I) renal biopsy and might be expected to present better functional recoverability after pyeloplasty.
\end{abstract}

Keywords: Ureteropelvic Junction Obstruction, Biopsy, Renal Pelvis, Hydronephrosis

\section{Background}

Ureteropelvic junction obstruction (UPJO) is a commonly encountered urinary tract abnormality, characterized by impairment of urine drainage, from the renal pelvis into the ureter, leading to hydronephrosis and obstructive changes in renal parenchyma. Although most cases are congenital, the problem may not become clinically evident until much later in life. The aim of treatment, in such cases, is to preserve the renal function and relieve symptoms by performing pyeloplasty, at the earliest. Diuretic renogram is the most commonly used imaging study to assess the renal function of the affected kidney, before and after surgery. Levels of biochemical markers, such as certain enzymes and proteins in urine, obtained from obstructed renal units (ORUs), have been used in predict- ing the final outcome of corrective surgery in patients with ureteropelvic obstruction. However, studies have shown that the biochemical markers have limited application and reliability, because of extremely variable results (1-6). Renal parenchymal thickness and pelvic diameter, measured by ultrasonography, also showed no consistent predictive value on functional outcome $(7,8)$. It was also found that clinical symptoms do not appear to affect renal function improvement after surgery. There are no statistical data to support that operating on asymptomatic patients is better than operating on symptomatic ones (9).

\section{Objectives}

None of the studies up to present is strong or conclusive enough to confirm the results in all cases. Studies have 
shown that the severity of histological changes, such as glomerulosclerosis, widening of Bowen's capsule, interstitial fibrosis and tubular atrophy, in the obstructed kidney, might influence on functional outcome after pyeloplasty (10-13).

\section{Patients and Methods}

The study was performed on 53 patients with hydronephrosis due to UPJO, over a period of 2 years, from December 2011 to December 2013.

Inclusion criteria:

Unilateral UPJO;

Normal functioning of contralateral kidney;

Differential renal function of obstructed kidney $>15 \%$.

Exclusion criteria:

Bilateral UPJO;

Secondary UPJO;

Infected hydronephrosis/Pyonephrosis;

Concomitant medical illness: diabetes, hypertension.

\subsection{Methods}

Apart from taking detailed history, analysis of genitourinary symptoms, general and focused neurological examination, local examination of genitalia and digital rectal examination were done in all patients, as per protocol. Routine urine analysis, comprising urine culture/sensitivity, complete blood count, routine blood tests, including liver function tests and renal function studies were performed in all patients. Radiological imaging studies by abdominal ultrasonography, intravenous pyelogram (IVP), and diethylene triamine pentaacetic acid (DTPA) were done in all cases. Differential renal function (DRF) and glomerular filtration rate (GFR) were evaluated by 99 mTc DTPA renogram. Patients were hydrated with normal saline $(10 \mathrm{mg} / \mathrm{kg}$ ) before injecting 99 mTc DTPA.

All patients with UPJO included in the study underwent open dismembered pyeloplasty and follow up DTPA renogram, which were done at the interval of 6 months and 12 months after surgical repair. During pyeloplasty, a small wedge of tissue, including full thickness cortical tissue from the lower pole of the affected kidney, was taken. All biopsy specimens were evaluated in a single laboratory using $5 \mu$ section and $\mathrm{H} \& \mathrm{E}$ staining. Histopathological analysis was done by one pathologist who had no knowledge about the clinical status of any patient, to avoid bias. Histopathological changes found in renal tissue were graded in terms of presence of severity of tubular and/or glomerular changes into three groups:
-Grade1-Normal glomerular and tubular structure(Figure 1).

- Grade 2 - Mild to moderate dilatation of collecting tubules and Bowen's space, minimal degree of interstitial inflammation (Figure 2).

- Grade 3 - Dysplastic glomeruli, marked interstitial fibrosis, tubular atrophy (Figure 3).

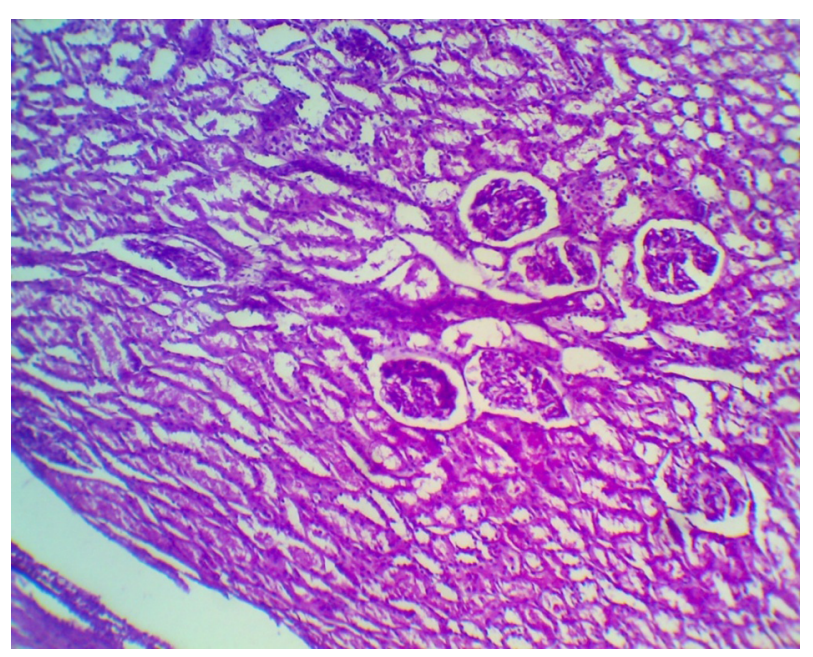

Figure 1. Grade I Histopathological Changes-Normal Tubular and Glomerular Structures

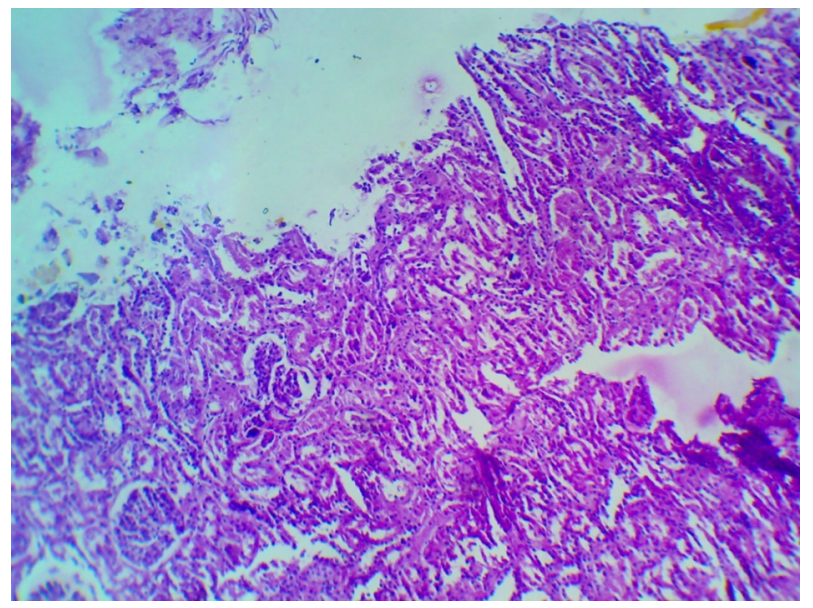

Figure 2. Grade 2, Histopathological Changes-Mild Dilatation of Collecting Tubules and Bowen's Space, Minimal Degree of Inflammation

If different grades were observed in a biopsy, the highest grade was assigned to that particular case. Patients were followed up to one year. The study was duly approved by the Institutional Ethics Committee. All patients 


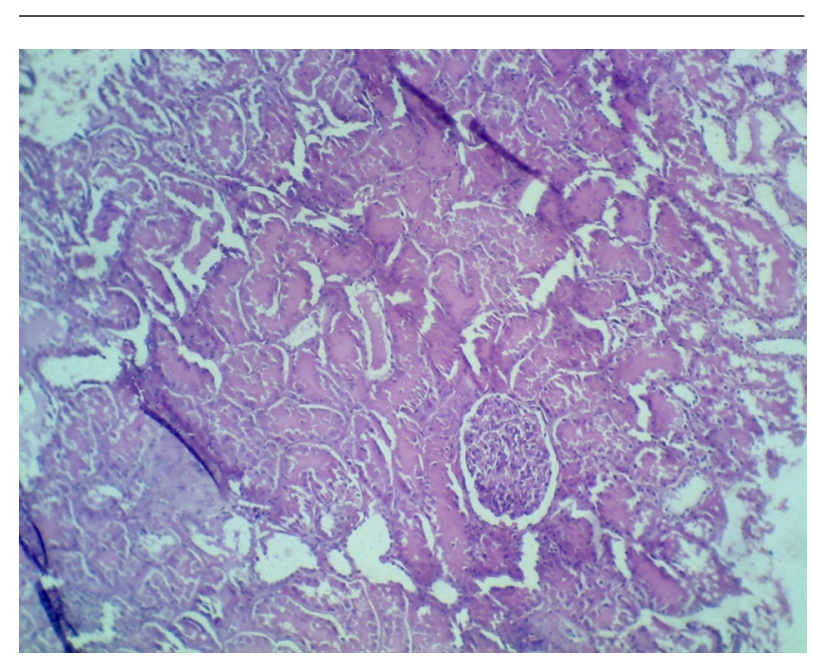

Figure 3. Grade 3, Histopathological Changes-Dysplastic Glomeruli Marked Interstitial Fibrosis, Tubular Atrophy

included in the study had given their informed consent. Statistical analysis of the test and outcome were compared by Fischer T-Test. A P $<0.05$ was considered statistically significant.

\section{Results}

The study group comprised 53 patients (aged 3 - 60 years), mean age $24.34 \pm 15.41$ years. Commonest age group was 10 - 19 years. Thirty eight (71.7\%) patients were male and $15(28.3 \%)$ were female. The affected kidney was on the right side in 15 (28.3\%) patients and on the left side in 38 (71.7\%) patients (Table 1). Low grade abdominal pain (66\%) was the commonest clinical presentation (Table 2 ). All patients underwent open dismembered pyeloplasty. The severity of histopathological changes in renal parenchyma was compared with the functional status of affected renal unit preoperatively and post-operatively (Table 3).

Table 1. Characteristics of Patients Included in the Study ${ }^{a, b}$

\begin{tabular}{lc}
\hline Total no. of Patients & $\mathbf{5 3}$ \\
\hline Median age, $\mathbf{y}$ & 24.34 \\
\hline Male & $38(71.7)$ \\
\hline Female & $15(28.3)$ \\
\hline Left UPJO & $38(71.7)$ \\
\hline Right UPJO & $15(28.3)$ \\
\hline${ }^{a}$ Abbreviation: UPJO, Ureteropelvic Junction Obstruction. \\
\hline
\end{tabular}

Table 2. Clinical Presentation ${ }^{a}$

\begin{tabular}{lc}
\hline Presentation & Total \\
\hline Abdominal pain & $35(66)$ \\
Renal lump & $3(5)$ \\
Incidental & $11(20)$ \\
Urinary tract infection & $3(5)$ \\
\hline
\end{tabular}

${ }^{\text {a }}$ Data are presented as No. (\%).

Table 3. Comparison of Grade of Renal Biopsy With Pre-Operative Differential Renal Function $^{\mathrm{a}, \mathrm{b}}$

\begin{tabular}{lccc}
\hline Grade & No. of Kidneys & DRF $<$ 35\% & DRF $>\mathbf{3 5 \%}$ \\
\hline I & 24 & $3(12.5)$ & $21(87.5)$ \\
II & 22 & $19(86.3)$ & $3(13.7)$ \\
III & 7 & $6(85.7)$ & $1(14.3)$ \\
\hline \multicolumn{2}{l}{ a Data are presented as No. (\%). } \\
b Abbreviations: DRF, differential renal function.
\end{tabular}

Out of 28 kidneys with DRF activity $<35 \%$ preoperatively, 25 (89.2\%) demonstrated grade 2 to grade 3 changes, while three kidneys had grade 1 changes. Of patients having DRF activity $>35 \%$ (25), 21 demonstrated grade 1 changes, three had grade 2 changes and only one had grade 3 histological changes. It is evident that $87.5 \%$ of patients of UPJO with grade 1 changes had good DRF ( $>35 \%$ ), while $89.2 \%$ of cases with grade 2 or grade 3 changes had poor $\operatorname{DRF}(<35 \%)$ preoperatively.

The ORUs having grade 1 changes in parenchyma had significant improvement in DRF $(\mathrm{P}<0.01)$. A slightly greater improvement in DRF was noted in the first 6 months in comparison to the 12 months value. On the other hand, kidneys having grade 2 or 3 histopathological changes in parenchyma showed insignificant improvement in $\operatorname{DRF}(\mathrm{P}>0.05)$ (Table 4).

\section{Discussion}

In hydronephrosis secondary to UPJO, significant renal histopathological changes are expected to occur, because of long standing obstruction in urinary drainage. Studies in animal model demonstrated histological changes in kidneys subjected to partial ureteral obstruction. Claesson et al. created partial ureteral obstruction in newborn rats, by burying the ureter in the psoas muscle (14). The effects of chronic partial obstruction were studied at the age of 6 weeks. Permanently partially obstructed kidneys had prominent papillary deformation that was associated with moderate widening of collecting duct and con- 
Table 4. Grade Wise Average Differential Renal Function at Different Stages ${ }^{\text {a,b }}$

\begin{tabular}{|c|c|c|c|c|c|}
\hline Grade & No. of Patients & Pre-Operative DRF & Post-Operative DRF 6 Months & Post-Operative DRF 12 Months & P Value \\
\hline $\mathbf{1}$ & 24 & $37.87 \pm 4.75$ & $41.55 \pm 4.05$ & $44.87 \pm 3.95$ & $<0.01$ \\
\hline 2 & 22 & $26.88 \pm 7.72$ & $29.70 \pm 7.47$ & $31.39 \pm 7.63$ & $>0.05$ \\
\hline 3 & 7 & $21.77 \pm 7.71$ & $22.74 \pm 7.96$ & $23.76 \pm 7.71$ & $>0.05$ \\
\hline
\end{tabular}

${ }^{\text {a }}$ Data are presented as Mean \pm SD.

b Abbreviations: DRF, differential renal function.

voluted tubules and focal inflammatory and degenerative changes. Steinhardt et al. performed nephrectomy in 20 cases of severe UPJO and the specimens were analyzed for histopathological changes. They showed that $75 \%$ had interstitial fibrosis, with inflammation, 70\% had glomerulosclerosis with inflammation, 30\% had medullary dysplasia and 15\% had glomerular cystic changes (15). Elder et al. showed that $79 \%$ of patients with DRF $>40 \%$ had mild alteration in renal histopathology, while $21 \%$ had severe alteration in renal histology. Elder et al. found that UPJ obstruction, with a differential function $<35 \%$ have a high probability of significant histological changes on biopsy (10). However, in our study, out of 24 kidneys, with grade 1 changes in renal parenchyma $87.5 \%(n=21)$ had DRF $>35 \%$ (mean DRF38.98 $\pm 3.90, \mathrm{P}<0.001$ ), while only three $(12.5 \%$ ) had $<35 \%$ DRF pre-operatively. Therefore, most of the obstructed renal units with DRF > 35\% have usually near normal renal biopsy.

One of the conflicting findings of our study was that four (12.5\%) patients, who had good DRF (> 35\%), had significant histological changes. This might be due to the error in differential function derived from the DTPA renogram. There is also the possibility of transient UPJO in utero that produced significant renal injury, which resolved subsequently, allowing normal maturation of renal function. However, further experimental studies are needed to justify this phenomenon.

On the other hand, it was found that three (12.5\%) patients with poor DRF $(<35 \%)$ had minimal or no histological changes on renal biopsy. Once again, this might be due to fallacious renal scan or there might be intermittent obstruction, which, at the time of study, showed poor DRF, although it had a good potential of functional recovery after release of obstruction. Another possibility is that the histopathological changes in the kidneys are heterogeneous and the part of renal tissue taken for biopsy might have reflected the portion of the kidney least affected by the UPJO.

\subsection{Outcome After Pyeloplasty}

Functional outcome of pyeloplasty is a matter of debate, since the pyeloplasty was first performed by Trendelenburg, in 1886 (16). Various studies have attempted to determine the functional outcome, with conflicting results. The clinician, as well as patient and parents, want to know the benefit of pyeloplasty in terms of recoverability of renal function and salvageability of kidney. One way to know it is by staging this treatment-putting stent or performing percutaneous nephrolithotomy (PCN) before definitive surgery and comparing results of radionuclide studies, done before and after diversion (17). However, this method is time consuming, with the attendant risk of stent dislodgement and infection. In this scenario, histopathological changes, occurring in renal parenchyma secondary to UPJO, might be useful in predicting the outcome of pyeloplasty in terms of renal function recoverability. Bhat et al. in their study, found that postoperative improvement in DRF is increased when the renal biopsy is suggestive of normal histology (18). Ortapamuk et al. have used the preoperative split renal function as the predicting factor for the outcome of adult pyeloplasty. According to them, the renal units with split renal function $<30 \%$ had very little chance of improvement after pyeloplasty (19). This study, however, showed that ORUs with no or minimal histopathological changes (grade I) had significant renal functional recoverability after 12 months of pyeloplasty. On the other hand, kidneys with significant changes (grade II and III) had minimal improvement in DRF, which was clinically insignificant $(\mathrm{P}>0.05)$. It was also found that a significant improvement is more likely in those with initial renal function $>35 \%$, $(\mathrm{P}<0.05)$. Although pyeloplasty is not very useful in kidneys having DRF $<35 \%$ or with significant histopathological changes in term of functional outcome, it is still important for relief of pain and to prevent complications, such as infection and urolithiasis.

One limitation of this study is that the functional recoverability has not been correlated with the age at which the operations were performed. Studies have shown that the pediatric age group has a superior functional recovery than adult patients, with similar preoperative DRF. Wagner 
M et al. in a retrospective study, have shown that the kidney is still salvageable in children with split function of even less than $10 \%$ (20). Therefore, the progressiveness with age or static evolution of renal parenchymal injury in cases of UPJO is still a matter of debate $(21,22)$.

Histopathological evaluation of renal parenchyma may be useful to provide an objective method of predicting the recovery of renal function after pyeloplasty. It would allow the comparison of the types of histological alteration, with the changes in DRF, in order to predict the final improvement potential of the affected renal unit, after successful corrective surgery. In the presence of severe pathological changes in renal biopsy, recoverability of renal function, despite anatomical success, in term of drainage, is significantly decreased. Patients having grade 1 histopathological changes have a high probability of improvement in DRF compared with those with grade 2 or grade 3 changes. The ORUs with DRF $>35 \%$ usually have minimal changes in renal biopsy and can be expected to get better functional recoverability. However, the DRF estimated on DTPA scan may be fallacious, particularly in grossly dilated renal pelvis and pyeloplasty, and should not be deferred when preservation of renal function is of concern. In this situation, renal biopsy may be useful, although it has certain disadvantages. First, image guided renal biopsy is an invasive procedure with risk of bleeding and infection, etc. Secondly, the histopathological changes may be heterogeneous and the biopsy from a small area may not be sufficient to provide an accurate histological estimate of the injury, sustained by the renal parenchyma, due to obstruction per se. It, therefore, needs further long term studies in a larger series of patients, in order to clearly define which kidneys are at risk for deterioration and to predict the improvement potential of affected kidneys.

\section{Acknowledgments}

We are thankful to the patients for allowing us to publish the data collected during their treatment.

\section{References}

1. Taha MA, Shokeir AA, Osman HG, Abd El-Aziz Ael A, Farahat SE. Obstructed versus dilated nonobstructed kidneys in children with congenital ureteropelvic junction narrowing: role of urinary tubular enzymes. J Urol. 2007;178(2):640-6. doi: 10.1016/j.juro.2007.04.006. [PubMed: 17574624].

2. Berry SM, Lecolier B, Smith RS, Bercau G, Dombrowski MP, Puder KS, et al. Predictive value of fetal serum beta 2-microglobulin for neonatal renal function. Lancet. 1995;345(8960):1277-8. [PubMed: 7746060].

3. Carr MC, Peters CA, Retik AB, Mandell J. Urinary levels of the renal tubular enzyme N-acetyl-beta-D-glucosaminidase in unilateral obstructive uropathy. J Urol. 1994;151(2):442-5. [PubMed: 8283554].
4. Huland H, Gonnermann D, Werner B, Possin U. A new test to predict reversibility of hydronephrotic atrophy after stable partial unilateral ureteral obstruction. J Urol. 1988;140(6):1591-4. [PubMed: 2903939].

5. Tataranni G, Farinelli R, Zavagli G, Logallo G, Farinelli A. Tubule recovery after obstructive nephropathy relief: the value of enzymuria and microproteinuria.J Urol. 1987;138(1):24-7. [PubMed: 2885428].

6. Chevalier RL, Goyal S, Thornhill BA. EGF improves recovery following relief of unilateral ureteral obstruction in the neonatal rat.J Urol. 1999;162(4):1532-6. [PubMed: 10492250].

7. Khalaf IM, Shokeir AA, El-Gyoushi FI, Amr HS, Amin MM. Recoverability of renal function after treatment of adult patients with unilateral obstructive uropathy and normal contralateral kidney: a prospective study. Urology. 2004;64(4):664-8. doi: 10.1016/j.urology.2004.05.018. [PubMed: 15491695].

8. Chavhan G, Daneman A, Moineddin R, Lim R, Langlois V, Traubici J. Renal pyramid echogenicity in ureteropelvic junction obstruction: correlation between altered echogenicity and differential renal function. Pediatr Radiol. 2008;38(10):1068-73. doi: 10.1007/s00247-0080943-5. [PubMed: 18633607].

9. Salem YH, Majd M, Rushton HG, Belman AB. Outcome analysis of pediatric pyeloplasty as a function of patient age, presentation and differential renal function. J Urol. 1995;154(5):1889-93. [PubMed: 7563377].

10. Elder JS, Stansbrey R, Dahms BB, Selzman AA. Renal histological changes secondary to ureteropelvic junction obstruction. $J$ Urol. 1995;154(2 Pt 2):719-22. [PubMed: 7609162].

11. Stock JA, Krous HF, Heffernan J, Packer M, Kaplan GW. Correlation of renal biopsy and radionuclide renal scan differential function in patients with unilateral ureteropelvic junction obstruction. J Urol. 1995;154(2 Pt 2):716-8. [PubMed: 7609161].

12. Huang WY, Peters CA, Zurakowski D, Borer JG, Diamond DA, Bauer $\mathrm{SB}$, et al. Renal biopsy in congenital ureteropelvic junction obstruction: evidence for parenchymal maldevelopment. Kidney Int. 2006;69(1):137-43. doi:10.1038/sj.ki.5000004. [PubMed: 16374434]

13. Erbagci A, Yag IF, Sarica K, Bakir K. Predictive value of renal histological changes for postoperative renal function improvement in children with congenital ureteropelvic junction stenosis. Int J Urol. 2002;9(6):279-84. [PubMed: 12110090].

14. Claesson G, Josephson S, Robertson B. Experimental partial ureteric obstruction in newborn rats. VII. Are the long term effects on renal morphology avoided by release of the obstruction?. J Urol. 1986;136(6):1330-4. [PubMed: 3773118].

15. Steinhardt GF, Ramon G, Salinas-Madrigal L. Glomerulosclerosis in obstructive uropathy.JUrol. 1988;140(5 Pt 2):1316-8. [PubMed: 3184311].

16. Poulakis V, Witzsch U, Schultheiss D, Rathert P, Becht E. [History of ureteropelvic junction obstruction repair (pyeloplasty). From Trendelenburg (1886) to the present]. Urologe A. 2004;43(12):1544-59. doi: 10.1007/s00120-004-0663-x. [PubMed: 15316607].

17. Gupta DK, Chandrasekharam VV, Srinivas M, Bajpai M. Percutaneous nephrostomy in children with ureteropelvic junction obstruction and poor renal function. Urology. 2001;57(3):547-50. [PubMed: 11248637].

18. Bhat GS, Maregowda S, Jayaram S, Siddappa S. Is renal biopsy a better predictor of the outcome of pyeloplasty in adult ureteropelvic junction obstruction?. Urology. 2012;79(2):321-5. doi: 10.1016/j.urology.2011.10.018. [PubMed: 22173181].

19. Ortapamuk H, Naldoken S, Tekdogan UY, Aslan Y, Atan A. Differential renal function in the prediction of recovery in adult obstructed kidneys after pyeloplasty. Ann Nucl Med. 2003;17(8):663-8. [PubMed: 14971608].

20. Wagner M, Mayr J, Hacker FM. Improvement of renal split function in hydronephrosis with less than $10 \%$ function. Eur J Pediatr Surg. 2008;18(3):156-9. doi: 10.1055/s-2008-1038445. [PubMed: 18484518].

21. Han SW, Lee SE, Kim JH, Jeong HJ, Rha KH, Choi SK. Does delayed operation for pediatric ureteropelvic junction obstruction cause histopathological changes?. J Urol. 1998;160(3 Pt 2):984-8. [PubMed: 9719259]. 
22. Zhang PL, Peters CA, Rosen S. Ureteropelvic junction obstruction: morphological and clinical studies. Pediatr Nephrol. 2000;14(8-
9):820-6. [PubMed: 10955936]. 